\title{
REDUCIBILITY AND BOSONIZATION OF PARASUPERSYMMETRIC AND ORTHOSUPERSYMMETRIC QUANTUM MECHANICS
}

\author{
C. QUESNE* AND N. VANSTEENKISTE \\ Physique Nucléaire Théorique et Physique Mathématique, \\ Université Libre de Bruxelles, Campus de la Plaine CP229, \\ Boulevard du Triomphe, B-1050 Brussels, Belgium \\ E-mail: cquesne@ulb.ac.be
}

\begin{abstract}
Order- $p$ parasupersymmetric and orthosupersymmetric quantum mechanics are shown to be fully reducible when they are realized in terms of the generators of a generalized deformed oscillator algebra and a $\mathbb{Z}_{p+1}$-grading structure is imposed on the Fock space. The irreducible components provide $p+1$ sets of bosonized operators corresponding to both unbroken and broken cases. Such a bosonization is minimal.
\end{abstract}

Running head: Reducibility and Bosonization

PACS: 03.65.Fd, 11.30.Pb

Keywords: quantum mechanics, supersymmetry, generalized deformed oscillator

${ }^{*}$ Directeur de recherches FNRS 


\section{Introduction}

Since the introduction of supersymmetric quantum mechanics by Witten [1], there has been a lot of interest in this field and its generalizations [2]. Among the latter, one may quote parasupersymmetric (PSSQM) [3, 4], orthosupersymmetric (OSSQM) [5], pseudosupersymmetric (PsSSQM) [6], and fractional supersymmetric quantum mechanics [7]. More recently, topological symmetries have also been introduced [8].

The standard way of realizing SSQM and its variants consists in combining bosons with fermions or more exotic particles. Since fermionic operators can be realized in terms of bosonic ones [9], it is straightforward to bosonize (i.e., to realize in terms of only boson-like operators without fermion-like ones) $N=2$ SSQM from the well-known Nicolai-Witten construction [1, 10] in terms of two commuting sets of independent operators. Some years ago, however, it was shown that SSQM can be bosonized in a different way [1]. Contrary to the former procedure, the latter is indeed a minimal bosonization in terms of a single bosonic degree of freedom. Such a result was obtained from a realization of SSQM in terms of generalized deformed bosonic oscillator operators [12] by imposing a $\mathbb{Z}_{2}$-grading structure on the deformed bosonic oscillator Fock space, which can be done, for instance, by restricting oneself to a Calogero-Vasiliev algebra [13]. In such a framework, SSQM turns out to be fully reducible and its irreducible components provide two sets of bosonized operators realizing either broken or unbroken SSQM.

An important open question is whether such results can be extended to SSQM variants. As far as the bosonization is concerned, some partial results were recently obtained in terms of generalized deformed oscillator algebras (GDOAs) $\mathcal{A}^{(\lambda)}(G(N))$ related to $C_{\lambda}$-extended oscillator ones, where $C_{\lambda}=\mathbb{Z}_{\lambda}$ denotes the cyclic group of order $\lambda(\lambda \in\{3,4,5, \ldots\})$ [14, 15] (see also [16] for a related work).

The aim of the present letter is to prove in full generality both the reducibility and the resultant bosonization of order- $p$ PSSQM and OSSQM, where in the former case we consider both the Rubakov-Spiridonov-Khare (RSK) approach [3] and the 
Beckers-Debergh (BD) one [4]. For such a purpose, we shall introduce some new matrix realizations of PSSQM and OSSQM in terms of GDOA generators and impose a $\mathbb{Z}_{p+1}$-grading structure on the corresponding Fock space. Restricting then ourselves to $\mathcal{A}^{(\lambda)}(G(N))$ with $\lambda=p+1$ will provide us with some interesting examples of bosonized operators.

\section{Generalized Deformed Oscillator Algebras}

There is an extensive literature on GDOAs to which we refer the reader (see e.g. [17] and references quoted therein). Here we shall only review some of their properties to be used in the next sections.

A GDOA may be defined as a nonlinear associative algebra $\mathcal{A}(G(N))$ generated by the operators $N=N^{\dagger}, a^{\dagger}$, and $a=\left(a^{\dagger}\right)^{\dagger}$, satisfying the commutation relations

$$
\left[N, a^{\dagger}\right]=a^{\dagger}, \quad[N, a]=-a, \quad\left[a, a^{\dagger}\right]=G(N)
$$

where $G(N)=[G(N)]^{\dagger}$ is some Hermitian function of $N$.

We restrict ourselves here to GDOAs possessing a bosonic Fock space representation. In the latter, we may write $a^{\dagger} a=F(N), a a^{\dagger}=F(N+1)$, where the structure function $F(N)=[F(N)]^{\dagger}$ is such that

$$
G(N)=F(N+1)-F(N)
$$

and is assumed to satisfy the conditions

$$
F(0)=0, \quad F(n)>0 \quad \text { if } n=1,2,3, \ldots
$$

The carrier space $\mathcal{F}$ of such a representation can be constructed from a vacuum state $|0\rangle$ (such that $a|0\rangle=N|0\rangle=0$ ) by successive applications of the creation operator $a^{\dagger}$. Its basis states

$$
|n\rangle=\left(\prod_{i=1}^{n} F(i)\right)^{-1 / 2}\left(a^{\dagger}\right)^{n}|0\rangle, \quad n=0,1,2, \ldots,
$$


where we set $\prod_{i=1}^{0} \equiv 1$, satisfy the relations $N|n\rangle=n|n\rangle, a^{\dagger}|n\rangle=\sqrt{F(n+1)}|n+1\rangle$, and $a|n\rangle=\sqrt{F(n)}|n-1\rangle$.

For $G(N)=I, F(N)=N$ and the algebra $\mathcal{A}(G(N))$ reduces to the standard (bosonic) oscillator algebra $\mathcal{A}(I)$, for which the creation and annihilation operators may be written as $a^{\dagger}=(x-\mathrm{i} P) / \sqrt{2}, a=(x+\mathrm{i} P) / \sqrt{2}$, where $P$ denotes the momentum operator $(P=-\mathrm{i} d / d x)$.

A $\mathbb{Z}_{\lambda}$-grading structure can be imposed on $\mathcal{F}$ by introducing a grading operator

$$
T=e^{2 \pi \mathrm{i} N / \lambda}, \quad \lambda \in\{2,3,4, \ldots\}
$$

which is such that

$$
T^{\dagger}=T^{-1}, \quad T^{\lambda}=I
$$

It has $\lambda$ distinct eigenvalues $q^{\mu}, \mu=0,1, \ldots, \lambda-1$, with corresponding eigenspaces $\mathcal{F}_{\mu} \equiv\{|k \lambda+\mu\rangle \mid k=0,1,2, \ldots\}$ such that $\mathcal{F}=\sum_{\mu=0}^{\lambda-1} \oplus \mathcal{F}_{\mu}$. Here $q$ denotes a $\lambda$-th root of unity, $q \equiv \exp (2 \pi \mathrm{i} / \lambda)$, and $|n\rangle=|k \lambda+\mu\rangle$ are the basis states (4). From (11), it results that $T$ satisfies the relations

$$
[N, T]=0, \quad a^{\dagger} T=e^{-2 \pi \mathrm{i} / \lambda} T a^{\dagger}, \quad a T=e^{2 \pi \mathrm{i} / \lambda} T a,
$$

expressing the fact that $N$ preserves the grade, while $a^{\dagger}$ (resp. a) increases (resp. decreases) it by one unit.

The operators

$$
P_{\mu}=\frac{1}{\lambda} \sum_{\nu=0}^{\lambda-1} e^{-2 \pi \mathrm{i} \mu \nu / \lambda} T^{\nu}, \quad \mu=0,1, \ldots, \lambda-1,
$$

project on the various subspaces $\mathcal{F}_{\mu}, \mu=0,1, \ldots, \lambda-1$, and therefore satisfy the relations

$$
P_{\mu}^{\dagger}=P_{\mu}, \quad P_{\mu} P_{\nu}=\delta_{\mu, \nu} P_{\mu}, \quad \sum_{\mu=0}^{\lambda-1} P_{\mu}=I
$$

in $\mathcal{F}$. As a consequence of $(\mathbb{7})$, they also fulfil the relations

$$
\left[N, P_{\mu}\right]=0, \quad a^{\dagger} P_{\mu}=P_{\mu+1} a^{\dagger}, \quad a P_{\mu}=P_{\mu-1} a
$$


where we use the convention

$$
P_{\mu^{\prime}}=P_{\mu} \quad \text { if } \mu^{\prime}-\mu=0 \bmod \lambda \text {. }
$$

As a special case of GDOA with a built-in $\mathbb{Z}_{\lambda}$-grading structure, we may consider the GDOA $\mathcal{A}^{(\lambda)}(G(N))$ associated with a $C_{\lambda^{-}}$extended oscillator algebra $\mathcal{A}_{\alpha_{0} \alpha_{1} \ldots \alpha_{\lambda-2}}^{(\lambda)}$, where the cyclic group $C_{\lambda}=\mathbb{Z}_{\lambda}$ is generated by $T$, i.e., $C_{\lambda}=\left\{T, T^{2}, \ldots, T^{\lambda-1}, T^{\lambda}=\right.$ I\} [14, 15].2] The GDOA $\mathcal{A}^{(\lambda)}(G(N))$ corresponds to the choice

$$
G(N)=I+\sum_{\mu=1}^{\lambda-1} \kappa_{\mu} T^{\mu}=I+\sum_{\mu=0}^{\lambda-1} \alpha_{\mu} P_{\mu}
$$

in Eq. (11). Here $\kappa_{\mu}, \mu=1,2, \ldots, \lambda-1$, are some complex parameters subject to the conditions $\kappa_{\mu}^{*}=\kappa_{\lambda-\mu}$, while $\alpha_{\mu}, \mu=0,1, \ldots, \lambda-1$, are some real ones constrained by $\sum_{\mu=0}^{\lambda-1} \alpha_{\mu}=0$. Hence the algebra depends upon $\lambda-1$ independent, real parameters, which may be chosen as $\alpha_{0}, \alpha_{1}, \ldots, \alpha_{\lambda-2}$.

The corresponding structure function can be expressed as

$$
F(N)=N+\sum_{\mu=0}^{\lambda-1} \beta_{\mu} P_{\mu}, \quad \beta_{0} \equiv 0, \quad \beta_{\mu} \equiv \sum_{\nu=0}^{\mu-1} \alpha_{\nu}, \quad \mu=1,2, \ldots, \lambda-1 .
$$

The existence of a Fock-space representation is guaranteed by the $\lambda-1$ constraints on the parameters $\sum_{\nu=0}^{\mu} \alpha_{\nu}>-\mu-1, \mu=0,1, \ldots, \lambda-2$, ensuring that $F(\mu)=$ $\mu+\beta_{\mu}>0$ for $\mu=1,2, \ldots, \lambda-1$.

The standard oscillator algebra $\mathcal{A}(I)$ is recovered in the limit $\alpha_{\mu} \rightarrow 0, \mu=0,1$, $\ldots, \lambda-1$.

\section{Order- $p$ Parasupersymmetric Quantum Me- chanics}

PSSQM of order $p$ is described in terms of parasupercharge operators $Q, Q^{\dagger}$, and of a parasupersymmetric Hamiltonian $\mathcal{H}$ satisfying the relations

$$
\begin{aligned}
Q^{p+1} & =0 \quad\left(\text { with } Q^{p} \neq 0\right), \\
{[\mathcal{H}, Q] } & =0
\end{aligned}
$$

${ }^{\mathrm{a}}$ In $\mathcal{A}_{\alpha_{0} \alpha_{1} \ldots \alpha_{\lambda-2}}^{(\lambda)}, T$ is considered as an operator independent of the remaining ones, so that Eqs. (6) and (7) (or alternatively (9) and (10) have to be postulated in addition to (1) and (12). The GDOA $\mathcal{A}^{(\lambda)}(G(N))$ corresponds to the realization (5) of $T$. 
and

$$
Q^{p} Q^{\dagger}+Q^{p-1} Q^{\dagger} Q+\cdots+Q Q^{\dagger} Q^{p-1}+Q^{\dagger} Q^{p}=2 p Q^{p-1} \mathcal{H}
$$

or

$$
\left[Q,\left[Q^{\dagger}, Q\right]\right]=2 Q \mathcal{H}
$$

according to whether one considers the RSK approach [3] or the BD one [4]. They also obey the Hermitian conjugated relations with $\left(Q^{\dagger}\right)^{\dagger}=Q$ and $\mathcal{H}^{\dagger}=\mathcal{H}$.

To start with, it is easy to get a $(p+1) \times(p+1)$-matrix realization of RSK PSSQM of the type

$$
Q=\sqrt{2} \sum_{i=1}^{p} f_{i}(N+1) a e_{i+1, i}, \quad Q^{\dagger}=\sqrt{2} \sum_{i=1}^{p} f_{i}(N) a^{\dagger} e_{i, i+1}, \quad \mathcal{H}=\sum_{i=1}^{p+1} H_{i} e_{i, i},
$$

in terms of the generators $N, a^{\dagger}$, a of a GDOA $\mathcal{A}(G(N))$. Here $f_{i}(N), i=1,2, \ldots$, $p$, are assumed to be some real functions of $N$ defined on the set $\{1,2,3, \ldots\}$, and $H_{i}, i=1,2, \ldots, p+1$, some $N$-dependent Hermitian operators, while $e_{i, j}$ denotes the $(p+1)$-dimensional matrix with entry 1 at the intersection of row $i$ and column $j$ and zeroes everywhere else. The ansatz (18) indeed automatically satisfies Eq. (14). Inserting it into the remaining two equations (15) and (16) leads to an explicit form for the Hamiltonians $H_{i}$,

$$
H_{i}=\frac{1}{p} \sum_{j=1}^{p} f_{j}^{2}(N+i-j) F(N+i-j), \quad i=1,2, \ldots, p+1
$$

in terms of the $p$ arbitrary functions $f_{i}(N), i=1,2, \ldots, p$, and of the structure function $F(N)$, solution of Eqs. (2) and (3).

It is worth mentioning that for $G(N)=I$ (corresponding to the standard oscillator algebra) and $f_{i}(N)=1, i=1,2, \ldots, p$, the parasupersymmetric Hamiltonian $\mathcal{H}$, as defined in (18) and (19), reduces to the RSK parasupersymmetric oscillator one, $\mathcal{H}_{\mathrm{osc}}^{\mathrm{RSK}}=\frac{1}{2}\left(P^{2}+x^{2}\right) \mathbb{I}-\operatorname{diag}\left(\frac{p}{2}, \frac{p}{2}-1, \ldots,-\frac{p}{2}+1,-\frac{p}{2}\right)$, where $\mathbb{I}$ denotes the $(p+1) \times(p+1)$ unit matrix. The corresponding charges $Q=\mathrm{i}(P-\mathrm{i} x) \sum_{i=1}^{p} e_{i+1, i}$, $Q^{\dagger}=-\mathrm{i}(P+\mathrm{i} x) \sum_{i=1}^{p} e_{i, i+1}$ only differ from the RSK ones by an irrelevant phase factor. 
The $(p+1) \times(p+1)$-matrix realization (18), (19) of RSK PSSQM can be diagonalized through a unitary transformation $U_{1}=\sum_{i, j=1}^{p+1} P_{i-j} e_{i, j}$, expressed in terms of the projection operators $P_{\mu}$ defined in (8) and (11) for $\lambda=p+1$. The results read

$$
\begin{aligned}
Q^{\prime} & \equiv U_{1} Q U_{1}^{\dagger}=\operatorname{diag}\left(Q_{0}, Q_{1}, \ldots, Q_{p}\right), \\
Q^{\prime \dagger} & \equiv U_{1} Q^{\dagger} U_{1}^{\dagger}=\operatorname{diag}\left(Q_{0}^{\dagger}, Q_{1}^{\dagger}, \ldots, Q_{p}^{\dagger}\right), \\
\mathcal{H}^{\prime} & \equiv U_{1} \mathcal{H} U_{1}^{\dagger}=\operatorname{diag}\left(\mathcal{H}_{0}, \mathcal{H}_{1}, \ldots, \mathcal{H}_{p}\right),
\end{aligned}
$$

where

$$
\begin{aligned}
Q_{\mu} & =\sqrt{2} \sum_{i=1}^{p} f_{i}(N+1) a P_{\mu+p+2-i}, \quad Q_{\mu}^{\dagger}=\sqrt{2} \sum_{i=1}^{p} f_{i}(N) a^{\dagger} P_{\mu+p+1-i}, \\
\mathcal{H}_{\mu} & =\sum_{i=1}^{p+1} H_{i} P_{\mu+p+2-i},
\end{aligned}
$$

for $\mu=0,1, \ldots, p$. Each of the $p+1$ sets of operators $\left\{Q_{\mu}, Q_{\mu}^{\dagger}, \mathcal{H}_{\mu}\right\}$ satisfies the RSK PSSQM algebra (14) - (16) and is written in terms of a single bosonic degree of freedom through the operators $N, a^{\dagger}, a$ of $\mathcal{A}(G(N))$. We have therefore proved that RSK PSSQM is fully reducible and, in addition, we have obtained a minimal bosonization thereof.

The eigenvalues $\mathcal{E}_{n}^{(\mu)}$ of the bosonized parasupersymmetric Hamiltonian $\mathcal{H}_{\mu}$, defined in (22), can be written as

$$
\begin{aligned}
\mathcal{E}_{k(p+1)+\nu}^{(\mu)}= & \frac{1}{p} \sum_{i=1}^{p} f_{i}^{2}[k(p+1)+\mu-i+1] F[k(p+1)+\mu-i+1] \\
& \text { if } \nu=0,1, \ldots, \mu, \\
\mathcal{E}_{k(p+1)+\nu}^{(\mu)}= & \frac{1}{p} \sum_{i=1}^{p} f_{i}^{2}[(k+1)(p+1)+\mu-i+1] F[(k+1)(p+1)+\mu-i+1] \\
& \text { if } \nu=\mu+1, \mu+2, \ldots, p,
\end{aligned}
$$

and therefore correspond to nonlinear spectra. At this stage, we may remark that since the sign of the structure function for negative integers is not fixed by condition (3), $\mathcal{E}_{\nu}^{(\mu)}$ may be positive, null, or negative for $\nu=0,1, \ldots, \mu$ and $\mu=0$, $1, \ldots, p-2$. We therefore recover here a well-known unsatisfactory feature of RSK PSSQM [3]. 
The results obtained so far may be illustrated by considering the GDOA $\mathcal{A}^{(p+1)}(G(N))$, defined in Sec. 2. This amounts to inserting the structure function (13) for $\lambda=p+1$ into Eqs. (19), (23), and (24). If $f_{i}^{2}(n), i=1,2, \ldots, p$, are chosen to be increasing functions of $n$, the ground state of the spectrum (23), (24) corresponds to $k=0, \nu=0,1, \ldots, \mu$, and is $(\mu+1)$-fold degenerate, while all the excited states are $(p+1)$-fold degenerate. Since $\mu$ may take any value in the set $\{0,1, \ldots, p\}$, the ground-state degeneracy may accordingly vary between 1 and $p+1$. As hereabove noted, it results from the constraints on the algebra parameters that the ground-state energy

$$
\begin{aligned}
\mathcal{E}_{0}^{(\mu)}= & \mathcal{E}_{1}^{(\mu)}=\cdots=\mathcal{E}_{\mu}^{(\mu)}=\frac{1}{p}\left[\sum_{i=1}^{\mu} f_{i}^{2}(\mu-i+1)\left(\mu-i+1+\beta_{\mu-i+1}\right)\right. \\
& \left.+\sum_{i=\mu+2}^{p} f_{i}^{2}(\mu-i+1)\left(\mu-i+1+\beta_{\mu+p+2-i}\right)\right]
\end{aligned}
$$

may have any sign except for $\mu=p-1$ or $\mu=p$, for which it is positive. It can be checked that $Q_{0}$ and $Q_{0}^{\dagger}$ have a vanishing action on $|0\rangle$, but that this is not true for $Q_{\mu}, Q_{\mu}^{\dagger}$ on $|0\rangle,|1\rangle, \ldots,|\mu\rangle$, when $\mu=1,2, \ldots$, or $p$. We conclude that unbroken (resp. broken) PSSQM corresponds to $\mu=0$ (resp. $\mu=1,2, \ldots$, or $p$ ).

In the special case where $f_{i}(N)=1, i=1,2, \ldots, p$, the bosonized parasupersymmetric Hamiltonian (22) associated with $\mathcal{A}^{(p+1)}(G(N))$ reduces to the operator (4.34) found in Ref. [15] and giving rise to a linear spectrum, while the bosonized parasupercharges (21) become the corresponding charges (up to an interchange of $Q_{\mu}$ and $Q_{\mu}^{\dagger}$, which leaves the PSSQM algebra invariant).

Let us now consider the case of BD PSSQM, defined by Eqs. (14), (15), and (17), and choose an ansatz similar to (18), except for a convenient renormalization of the functions $f_{i}(N)$,

$$
\begin{aligned}
Q & =\sum_{i=1}^{p}[i(p-i+1)]^{1 / 2} f_{i}(N+1) a e_{i+1, i}, \quad Q^{\dagger}=\sum_{i=1}^{p}[i(p-i+1)]^{1 / 2} f_{i}(N) a^{\dagger} e_{i, i+1}, \\
\mathcal{H} & =\sum_{i=1}^{p+1} H_{i} e_{i, i} .
\end{aligned}
$$

It is easy to show that Eqs. (15) and (17) are satisfied provided

$$
H_{i}=f_{1}^{2}(N+i-1) F(N+i-1), \quad i=1,2, \ldots, p+1,
$$


and, in addition, the functions $f_{i}(N)$ fulfil the conditions

$$
f_{i}(N) \sqrt{F(N)}=\epsilon_{i}(N) f_{1}(N+i-1) \sqrt{F(N+i-1)}, \quad i=2,3, \ldots, p
$$

where $\epsilon_{i}^{2}(N)=1$. The general solution of (28) can be written as

$$
f_{i}(N)=\epsilon_{i}(N) g(N+i-1)\left(\prod_{\substack{j=1 \\ j \neq i}}^{p} F(N+i-j)\right)^{1 / 2}, \quad i=1,2, \ldots, p,
$$

with $\epsilon_{1}(N) \equiv 1$ and $g(N)$ an arbitrary real function of $N$. Hence Eq. (27) finally becomes

$$
H_{i}=g^{2}(N+i-1) \prod_{j=1}^{p} F(N+i-j), \quad i=1,2, \ldots, p+1 .
$$

As in the RSK case, the $(p+1)$-dimensional matrix realization of BD PSSQM, given in (26), (29), and (30), can be diagonalized through the unitary transformation $U_{1}$. The bosonized parasupercharges and parasupersymmetric Hamiltonian are still given by Eqs. (21) and (22) (up to the renormalization $\sqrt{2} f_{i}(N) \rightarrow[i(p-i+$ 1) $]^{1 / 2} f_{i}(N)$ ), where $f_{i}(N)$ and $H_{i}$ are now expressed in the form (29) and (30), respectively.

The eigenvalues $\mathcal{E}_{n}^{(\mu)}$ of $\mathcal{H}_{\mu}$ can be written as

$$
\begin{aligned}
\mathcal{E}_{k(p+1)+\nu}^{(\mu)}= & g^{2}[k(p+1)+\mu] \prod_{i=1}^{p} F[k(p+1)+\mu-i+1] \\
& \text { if } \nu=0,1, \ldots, \mu \\
\mathcal{E}_{k(p+1)+\nu}^{(\mu)}= & g^{2}[(k+1)(p+1)+\mu] \prod_{i=1}^{p} F[(k+1)(p+1)+\mu-i+1] \\
& \text { if } \nu=\mu+1, \mu+2, \ldots, p .
\end{aligned}
$$

Here we may remark that contrary to what happens in the RSK case, no eigenvalue can be negative because every time a structure function with negative argument appears in (31), it is multiplied by $F(0)$, which is vanishing according to (3). We therefore recover a property of BD PSSQM [4].

For the GDOA $\mathcal{A}^{(p+1)}(G(N))$ and an increasing function $g^{2}(n)$, the properties of the spectrum are similar to those obtained hereabove in the RSK case, except that 
now the ground-state energy $\mathcal{E}_{0}^{(\mu)}=\mathcal{E}_{1}^{(\mu)}=\cdots=\mathcal{E}_{\mu}^{(\mu)}$ vanishes for $\mu=0,1, \ldots, p-1$ and is equal to $g^{2}(p) \prod_{i=1}^{p}\left(p-i+1+\beta_{p-i+1}\right)>0$ for $\mu=p$. It can be checked that the former (resp. latter) case corresponds to unbroken (resp. broken) PSSQM. This agrees with the detailed study of $p=2$ parasuperspectra carried out in Ref. [4].

Had we chosen $f_{i}(N)=1, i=1,2, \ldots, p$, from the very beginning in Eq. (26), the conditions (28) would lead to some constraints on the structure function. For $p=2$, for instance, we would get a single constraint $F(N)=F(N+1)$. For the GDOA $\mathcal{A}^{(p+1)}(G(N))$, it gives rise to the conditions $\alpha_{\mu}=-1, \mu=0,1,2$, which are incompatible with the existence of a Fock space representation (see also Ref. [14]). We conclude that for the proof of the reducibility of BD PSSQM and its resultant bosonization, the presence of nontrivial functions $f_{i}(N)$ in (26) plays a crucial role.

It should be noted that the BD parasupersymmetric oscillator Hamiltonian [ [4] cannot be seen as a special case of the matrix realization (26) when $G(N) \rightarrow I$. There actually exists another $(p+1) \times(p+1)$-matrix realization of BD PSSQM in terms of $\mathcal{A}(G(N))$ generators that has this property: instead of having a everywhere below the principal diagonal in $Q$, it has $a^{\dagger}$ and $a$ appearing there in turn. Such a realization is however not reducible along the lines of the present work.

\section{Order-p Orthosupersymmetric Quantum Me- chanics}

OSSQM of order $p$ is formulated in terms of an orthosupersymmetric Hamiltonian $\mathcal{H}$ and $p$ pairs of orthosupercharge operators $Q_{i}, Q_{i}^{\dagger}, i=1,2, \ldots, p$, satisfying the relations

$$
\begin{aligned}
Q_{i} Q_{j} & =0, \\
{\left[\mathcal{H}, Q_{i}\right] } & =0, \\
Q_{i} Q_{j}^{\dagger}+\delta_{i, j} \sum_{k=1}^{p} Q_{k}^{\dagger} Q_{k} & =2 \delta_{i, j} \mathcal{H},
\end{aligned}
$$

and their Hermitian conjugates with $\left(Q_{i}^{\dagger}\right)^{\dagger}=Q_{i}$ and $\mathcal{H}^{\dagger}=\mathcal{H}$, where $i, j$ run over $1,2, \ldots, p[5]$. 
A $(p+1) \times(p+1)$-matrix realization in terms of $\mathcal{A}(G(N))$ generators can be found by setting

$$
Q_{i}=\sqrt{2} f_{i}(N+i) a^{i} e_{1, i+1}, \quad Q_{i}^{\dagger}=\sqrt{2} f_{i}(N)\left(a^{\dagger}\right)^{i} e_{i+1,1}, \quad \mathcal{H}=\sum_{i=1}^{p+1} H_{i} e_{i, i}
$$

where $f_{i}(N), i=1,2, \ldots, p$, are some real functions of $N$, defined on the set $\{i, i+1, \ldots\}$, and $H_{i}, i=1,2, \ldots, p+1$, are some $N$-dependent Hermitian operators. One indeed finds that Eqs. (33) - (35) are satisfied provided

$$
H_{i}=f_{p}^{2}(N+p+1-i) \prod_{j=1}^{p} F(N+j+1-i), \quad i=1,2, \ldots, p+1,
$$

and

$$
f_{i}(N)=\epsilon_{i}(N) f_{p}(N+p-i)\left(\prod_{j=1}^{p-i} F(N+j)\right)^{1 / 2}, \quad i=1,2, \ldots, p,
$$

where $\epsilon_{i}^{2}(N)=1$.

The matrices (36) can be reduced by means of the unitary transformation $U_{2}=$ $\sum_{i, j=1}^{p+1} P_{i+j-1} e_{i, j}$, where the $P_{\mu}$ 's are again the projection operators (8), (11) for $\lambda=p+1$. As a consequence, we obtain $p+1$ sets of bosonized orthosupercharges and orthosupersymmetric Hamiltonian

$$
\begin{aligned}
Q_{i \mu} & =\sqrt{2} f_{i}(N+i) a^{i} P_{\mu+i+1}, \quad Q_{i \mu}^{\dagger}=\sqrt{2} f_{i}(N)\left(a^{\dagger}\right)^{i} P_{\mu+1}, \\
\mathcal{H}_{\mu} & =\sum_{i=1}^{p+1} H_{i} P_{\mu+i},
\end{aligned}
$$

where $\mu=0,1, \ldots, p$, and $H_{i}, f_{i}(N)$ are given in Eqs. (37), (38), respectively.

The eigenvalues $\mathcal{E}_{n}^{(\mu)}$ of $\mathcal{H}_{\mu}$, defined in (40), can be written as

$$
\begin{aligned}
\mathcal{E}_{k(p+1)+\nu}^{(\mu)}= & f_{p}^{2}[k(p+1)+\mu] \prod_{i=1}^{p} F[k(p+1)+\mu-p+i] \\
& \text { if } \nu=0,1, \ldots, \mu \\
\mathcal{E}_{k(p+1)+\nu}^{(\mu)}= & f_{p}^{2}[(k+1)(p+1)+\mu] \prod_{i=1}^{p} F[(k+1)(p+1)+\mu-p+i] \\
& \text { if } \nu=\mu+1, \mu+2, \ldots, p,
\end{aligned}
$$

and therefore correspond to nonlinear spectra again. As in the case of BD PSSQM, any structure function with negative argument is multiplied by $F(0)$, hence $\mathcal{E}_{\nu}^{(\mu)}$ is always nonnegative. 
When we choose the GDOA $\mathcal{A}^{(p+1)}(G(N))$ and an increasing function $f_{p}^{2}(n)$, we find that for $\mu=0,1, \ldots, p-1$, the orthosupersymmetry is unbroken and the ground state, corresponding to $k=0, \nu=0,1, \ldots, \mu$, has a vanishing energy and a degeneracy $\mu+1$. On the contrary, for $\mu=p$, the orthosupersymmetry is broken and the $(p+1)$-fold degenerate ground state has a positive energy, given by $\mathcal{E}_{0}^{(p)}=\mathcal{E}_{1}^{(p)}=\cdots=\mathcal{E}_{p}^{(p)}=f_{p}^{2}(p) \prod_{i=1}^{p}\left(i+\beta_{i}\right)$. In both cases, all the excited states have a degeneracy $p+1$. Such results are in accordance with those of Khare et al. [5].

As for BD PSSQM, the presence of nontrivial functions $f_{i}(N)$ in (36) is essential to prove the reducibility and bosonization of OSSQM in full generality. Setting $f_{i}(N)=1, i=1,2, \ldots, p$, in (38) would indeed lead to some restrictions on the structure function.

The bosonized operators (39), (40) differ from those previously obtained for $p=2$ and linear spectra, which are linear in the creation and annihilation operators [15]. The latter may actually be generalized to nonlinear spectra by introducing some $N$-dependent coefficients and they may be derived by reducing some appropriate $3 \times 3$-matrix realization of $p=2$ OSSQM. A straightforward extension to higher values of $p$ however proves impossible. To get round this difficulty, it is necessary to consider powers of the creation and annihilation operators, as we did in (36) and (39). As a consequence, the orthosupersymmetric oscillator Hamiltonian [5] cannot be retrieved in the limit $G(N) \rightarrow I$.

\section{Conclusion}

In the present work, we did prove that both versions of order- $p$ PSSQM, as well as order- $p$ OSSQM, are fully reducible when we realize them in terms of GDOA generators and impose a $\mathbb{Z}_{p+1}$-grading on the Fock space. As a consequence, we did get a minimal bosonization of such SSQM variants in terms of a single bosonic degree of freedom. Similar results have been obtained elsewhere in the case of 
PsSSQM [18]. It is worth stressing that everything remains true in the limit $G(N) \rightarrow$ $I$, corresponding to the standard bosonic oscillator algebra.

In the cases of BD PSSQM anf of OSSQM, our results were obtained at the expense of considering Hamiltonians that in the limit $G(N) \rightarrow I$, contain powers of $P^{2}$ and would therefore perhaps more appropriately be called 'quasi-Hamiltonians'. In addition, the OSSQM charges contain powers of $P$ in the same limit. Since such features are characteristic of higher-derivative SSQM [19] and $\mathcal{N}$-fold SSQM [20], the existence of connections with these theories is an interesting open question, which we hope to discuss in a near future. 


\section{References}

[1] E. Witten, Nucl. Phys. B188, 513 (1981); B202, 253 (1982).

[2] F. Cooper, A. Khare and U. Sukhatme, Phys. Rep. 251, 267 (1995); B. Bagchi, Supersymmetry in Quantum and Classical Mechanics (Chapman and Hall / CRC, Florida, 2000).

[3] V. A. Rubakov and V. P. Spiridonov, Mod. Phys. Lett. A3, 1337 (1988); A. Khare, J. Math. Phys. 34, 1277 (1993).

[4] J. Beckers and N. Debergh, Nucl. Phys. B340, 767 (1990); Z. Phys. C51, 519 (1991); J. Phys. A26, 4311 (1993).

[5] A. Khare, A. K. Mishra and G. Rajasekaran, Int. J. Mod. Phys. A8, 1245 (1993).

[6] J. Beckers and N. Debergh, Int. J. Mod. Phys. A10, 2783 (1995).

[7] S. Durand, Phys. Lett. B312, 115 (1993); Mod. Phys. Lett. A8, 1795 (1993).

[8] A. Mostafazadeh and K. Aghababaei Samani, Mod. Phys. Lett. A15, 175 (2000); K. Aghababaei Samani and A. Mostafazadeh, Nucl. Phys. B595, 467 (2001).

[9] S. Naka, Prog. Theor. Phys. 59, 2107 (1978).

[10] H. Nicolai, J. Phys. A9, 1497 (1976).

[11] M. S. Plyushchay, Ann. Phys. (N.Y.) 245, 339 (1996); Mod. Phys. Lett. A11, 397 (1996); J. Beckers, N. Debergh and A. G. Nikitin, Int. J. Theor. Phys. 36, 1991 (1997).

[12] D. Bonatsos and C. Daskaloyannis, Phys. Lett. B307, 100 (1993).

[13] M. A. Vasiliev, Int. J. Mod. Phys. A6, 1115 (1991). 
[14] C. Quesne and N. Vansteenkiste, Phys. Lett. A240, 21 (1998); Helv. Phys. Acta 72, 71 (1999).

[15] C. Quesne and N. Vansteenkiste, Int. J. Theor. Phys. 39, 1175 (2000).

[16] M. Daoud and M. Kibler, "On fractional supersymmetric quantum mechanics: The fractional supersymmetric oscillator," Preprint math-ph/0101009; "On two approaches to fractional supersymmetric quantum mechanics," Preprint mathph/0110031.

[17] J. Katriel and C. Quesne, J. Math. Phys. 37, 1650 (1996); C. Quesne and N. Vansteenkiste, J.Phys. A28, 7019 (1995); Helv. Phys. Acta 69, 141 (1996).

[18] C. Quesne and N. Vansteenkiste, "Pseudosupersymmetric quantum mechanics: General case, orthosupersymmetries, reducibility, and bosonization," Preprint math-ph/0203035.

[19] A. A. Andrianov, M. V. Ioffe and V. P. Spiridonov, Phys. Lett. A174, 273 (1993); A. A. Andrianov, M. V. Ioffe, F. Cannata and J.-P. Dedonder, Int. J. Mod. Phys. A10, 2683 (1995); A. A. Andrianov, M. V. Ioffe and D. N. Nishnianidze, Theor. Math. Phys. 104, 1129 (1995).

[20] H. Aoyama, M. Sato, T. Tanaka and M. Yamamoto, Phys. Lett. B498, 117 (2001); H. Aoyama, M. Sato and T. Tanaka, ibid. B503, 423 (2001); Nucl. Phys. B619, 105 (2001). 